\title{
Questes
}

\section{Grivoiserie, pornographie, scatologie : introduction}

\author{
Marie Dupuy et Mathilde Grodet
}

\section{(2) OpenEdition}

\section{Journals}

\section{Édition électronique}

URL : http://journals.openedition.org/questes/2636

DOI : 10.4000/questes.2636

ISSN : 2109-9472

\section{Éditeur}

Les Amis de Questes

\section{Édition imprimée}

Date de publication : 15 juin 2011

Pagination : 10-34

ISSN : 2102-7188

\section{Référence électronique}

Marie Dupuy et Mathilde Grodet, « Grivoiserie, pornographie, scatologie : introduction », Questes [En ligne], 21 | 2011, mis en ligne le 15 janvier 2014, consulté le 22 septembre 2020. URL : http:// journals.openedition.org/questes/2636 ; DOI : https://doi.org/10.4000/questes.2636 


\section{Grivoiserie, pornographie, scatologie Introduction}

\section{Marie DUPUY et Mathilde GRODET}

«-Quel est le mot le plus poilu du psautier?

- Conculcavit!»

Le lecteur moderne pourrait être surpris, sinon choqué, de lire de telles plaisanteries dans un recueil de divertissements destiné à la société huppée... C'est pourtant dans un ouvrage semblable, rassemblant des devinettes prisées de l'aristocratie du $\mathrm{XV}^{\mathrm{e}}$ siècle, que Bruno Roy a découvert cette «demande joyeuse $»^{1}$. Il faut reconnaître que nous continuons de nous faire du Moyen Âge une image contrastée, entre la vision éthérée de la civilisation qui inventa l'amour courtois et le cliché sombre d'une époque souvent associée à l'obscurantisme. Se poser la question de la place, ou du moins de l'existence, des notions de grivoiserie, de pornographie et de scatologie au Moyen Âge doit nous obliger à nous dépouiller de nos préjugés pour approcher autant que faire se peut les mentalités médiévales.

Grivoiserie, pornographie, scatologie : voici trois notions qui ont en commun une certaine idée de transgression liée aux représentations du corps, et en particulier de ce que l'on définit depuis Mikhaïl Bakhtine comme le domaine $\mathrm{du}$ «bas corporel $»^{2}$. Le terme «scatologie» désigne les propos et les écrits grossiers traitant des excréments. Nous remarquons

\footnotetext{
Devinettes françaises $d u$ Moyen Âge, Bruno Roy (éd.), Montréal/Paris, Bellarmin/Vrin, 1977, p. 88. Il s'agit de la devinette $\mathrm{n}^{\circ} 166$ : «Quel est le plus velu mot de tout le grant psaultier? - C'est conculcavit. ». Elle est traduite dans l'ouvrage du même auteur Une Culture de l'équivoque, Montréal/Paris, Champion-Slatkine/Presses de l'Université de Montréal, 1992, chap. VI «Les voies de l'humour érotique » p. 89-100 (p. 91).

2 Cf. Mikhaïl BAKHTine, L'Euvre de François Rabelais et la culture populaire au Moyen Âge et sous la Renaissance, Paris, Gallimard, 1990 ( $1^{\text {ère }}$ éd. Gallimard 1970/1 ${ }^{\text {ère }}$ éd. russe 1965).
} 
que les dictionnaires insistent sur le caractère verbal de la scatologie, alors qu'il existe d'autres modes de représentations, notamment des sculptures, des enluminures... ${ }^{3}$. Les mots « grivoiserie » et «pornographie » renvoient tous deux à l'évocation de la sexualité, mais se distinguent de l'érotisme, qui est la recherche et l'expression esthétique de la sensualité. La grivoiserie évoque une gaieté licencieuse, de nature libidineuse. Elle se différencie de l'érotisme par sa légèreté et parfois sa dimension comique. Enfin, la pornographie est la représentation d'objets sexuels sans préoccupation artistique et avec l'intention délibérée de provoquer l'excitation du public auquel elle est destinée. Contrairement à l'érotisme qui peut avoir une finalité artistique et fonctionne sur l'ambiguïté, la pornographie se caractérise par son univocité et souvent par sa dimension vénale. Ces trois termes, « grivoiserie », « pornographie » et « scatologie », sont à peu près contemporains. Ils apparaissent au XIX ${ }^{\mathrm{e}}$ siècle, bien après la période qui nous occupe. S'agit-il de mots récents, mais qui recouvrent des réalités qui existaient déjà à l'époque médiévale ? Ou sont-ils le reflet de la morale pudibonde de ce $\mathrm{XIX}^{\mathrm{e}}$ siècle finissant ? Nous laissons aux différents intervenants le soin d'éclaircir ce point. Notons simplement que les hommes du Moyen Âge préféraient l'adjectif « gaillard » à «grivois » et recouraient plus volontiers à des catégories religieuses comme la luxure ou la fornication.

Grivoiserie, pornographie et scatologie relèvent donc de l'obscénité, concept instable qui désigne ce qui n'est pas montrable, ce que l'on doit tenir hors de la scène parce qu'il offense la pudeur et la décence ${ }^{4}$, mais qui ne relève d'aucune catégorie esthétique, philosophique, sociologique...

\footnotetext{
${ }^{3}$ Cf. illustrations 1 et 2 , p. 31 .

${ }^{4}$ Bien que le lien étymologique parfois établi entre «obscène » et «scène » ne soit pas philologiquement correct, l'obscénité est généralement pensée en termes de représentation. Est obscène non pas ce que l'on ne peut pas faire, mais ce que l'on ne peut pas faire en public.
} 
L'obscénité dépend finalement de la subjectivité de chacun et des conventions de chaque époque. Il y a donc une réelle difficulté à appréhender, mille ans après, ce qui fut effectivement transgressif ou non pour les hommes du Moyen Âge. Comment interpréter des représentations du bas corporel qui peuvent nous surprendre aujourd'hui sans tomber dans l'anachronisme?

\section{Le bas corporel au Moyen Âge : mise en perspective historique}

Examiner les notions de grivoiserie, de pornographie et de scatologie implique de s'interroger sur la perception du bas corporel au Moyen Âge, à une époque où le corps est problématique puisqu'il est à la fois déprécié et valorisé, sacralisé et dévalué 5 .

\section{Le haut et le bas}

Si la descente de l'âme dans le corps n'est pas une déchéance pour la première patristique, dès les $\mathrm{II}^{\mathrm{e}}$ et $\mathrm{III}^{\mathrm{e}}$ siècles l'incarnation devient, suivant les théories platoniciennes, une dégradation. Le corps n'est pourtant jamais totalement indigne puisqu'il participe de la résurrection, qui ne peut être complète sans lui et, après Origène, on tend à l'admirer car il est une création divine : l'homme est à l'image de Dieu, entièrement et à la fois âme et corps. Ce dernier n'est donc jamais mauvais en soi, c'est la chair ${ }^{6}$

\footnotetext{
${ }^{5}$ Cf. Jacques Le Goff et Nicolas TruOng, Une Histoire du corps au Moyen Âge, Paris, Liana Levi, 2003, p. 35-96.

${ }^{6} \mathrm{La}$ «chair » n'est pas synonyme de « corps ». Le mot désigne la nature humaine tout entière en tant qu'elle s'oppose à Dieu et à la grâce : c'est par son élément matériel que l'homme diffère le plus du divin. Voir l'article "Chair» dans le Dictionnaire de spiritualité ascétique et mystique. Doctrine et histoire, fondé par M. VILLER, F. Cavalera, J. De Guibert et A. Rayez, continué par A. Derville, P. Lamarche et A. Solignac, Paris, Beauchesne, 1932-1995, t. II. D’autre part, Rémi Brague montre que la chair n'est pas toujours perçue négativement, en particulier chez saint Bernard: cf. Rémi Brague, Au Moyen du Moyen Âge. Philosophies médiévales en chrétienté, judaïsme et islam, Chatou, Les Éditions de la Transparence, 2006, $2^{\text {ème }}$ partie, chap. II «Un modèle médiéval de la subjectivité : la chair», p. 126-128. Cf. aussi Jacques LE
} 
qui l'est. La sexualité, relevant de la chair, est la forme la plus aboutie de la dégradation du corps. Elle appartient au «bas corporel », pour reprendre l'expression de Mikhaïl Bakhtine qui renvoie au clivage spatial privilégié par l'Occident médiéval, celui du haut et du bas.

Alors que toute l'anthropologie chrétienne vise à une élévation spirituelle, le bas du corps est associé à l'ordure de la boue et de l'immondice et replace l'homme dans la sphère de l'animalité. Ainsi, pour Bernard de Clairvaux, la sexualité et la concupiscence sont du domaine animal et la limite que l'on franchit en transgressant les lois morales est celle qui permet de définir ce qui est humain et ce qui est bestial :

Quid mirum si similem sortimur exitum, qui et similem habemus introitum? Unde enim hominibus, nisi de similitudine bestiali, ille tam intemperans ardor in coitu, tam immoderatus dolor in partu ? Ita homo in conceptu et ortu, in vita et morte, comparatus est jumentis insipientibus, et similis factus est illis (Ps. 48, 13). ${ }^{7}$

\section{Nudité et pudeur}

Le corps primitif, dans sa nudité, était un corps pur. Mais, chargé du poids de la chair, il se couvre et se cache ; la nudité et sa représentation deviennent problématiques en même temps que le corps.

Dans l'Antiquité, des représentations que nous pourrions qualifier de pornographiques semblent bel et bien exister: on trouve déjà les images réalistes d'organes génitaux, les fantasmes, les détails exagérés, la variété

GofF, L'Imaginaire médiéval, in Un autre Moyen Âge, Paris, Gallimard, 1999, «Quarto », p. 421-770 et en particulier le chapitre III, traitant du corps, p. 553-578.

7 «Quoi d'étonnant si nous avons pour lot une sortie de la vie semblable à celle des bêtes, nous qui avons aussi une entrée semblable à la leur ? D'où viennent aux hommes, sinon de leur ressemblance avec les bêtes, une ardeur si immodérée dans l'acte sexuel, une douleur si vive dans l'enfantement ? C'est ainsi que l'“homme", dans sa conception et dans sa naissance, dans sa vie et dans sa mort, "a été assimilé aux bêtes sans raison et leur est devenue semblable" », BERnARD DE ClairvauX, Sermon, 82, 5, in Sermons sur le Cantique des Cantiques. $t$. V. Sermons 69-86, texte latin de J. LECLERCQ, H. RochaIS et Ch. H. TALbot, introd. et notes de Paul VerdeYen et trad. de Raffaele FASSETTA, Paris, Le Cerf, « Sources chrétiennes », 511, 2007, p. 333 et 335. 
des positions, la violence et l'humiliation des femmes ${ }^{8}$. L'usage en est généralement domestique; les fins de telles représentations étaient didactiques autant qu'incitatives, et souvent destinées aux femmes, dévouées au service du plaisir masculin ${ }^{9}$. De façon générale, la représentation du nu est presque topique dans l'Antiquité. Pourtant il semble que la pudeur soit de mise dans la sphère privée et Paul Veyne nous met en garde: "l'antique nudité, le rêve de sensualité païenne et de candide nature, sont des mythes », dit-il, insistant sur l'écart entre imaginaire et mimesis ${ }^{10}$.

Au Moyen Âge, en revanche, les images de corps dénudés se font plus rares et, en renversant l'observation de Paul Veyne, nous pouvons nous demander si ces représentations d'amants pudiquement recouverts d'un drap renvoient à une quelconque réalité. Il semble bien qu'au quotidien le corps soit rarement donné à voir dans sa nudité, mais le vêtement est considéré comme une seconde peau, à tel point que se montrer en chemise, c'est se montrer quasi $\mathrm{nu}^{11}$. Chez les plus pauvres cependant, il y a peu de linge de corps, ce qui peut laisser entendre une certaine familiarité avec le corps dénudé ${ }^{12}$. La question est d'importance aussi dans

\footnotetext{
${ }^{8}$ Cf. Harvey Alan SHAPIRO, «Eros in love : Pederasty and Pornography in Greece », in Amy Richlin (dir.), Pornography and Representation in Greece and Rome, NewYork/Oxford/Toronto, Oxford University Press, 1992, p. 53-72.

${ }^{9}$ Voir en particulier à ce sujet Laurent MARTIN, « Jalons pour une histoire culturelle de la pornographie en occident », Le Temps des médias, 2003/1 n¹, p. 10-30.

10 Paul VEYNE, «La famille et l'amour sous le Haut-Empire romain », Annales. Économies, Sociétés, Civilisations, 33 (1978), n¹, p. 54.

${ }^{11}$ Cf. Romaine Wolf-Bonvin, «Un vêtement sans l'être : la chemise », in Le Nu et le vêtu au Moyen Âge (XII ${ }^{e}$-XIII siècles). Actes du $25^{\mathrm{e}}$ colloque du CUER MA, 2-3-4 mars 2000, Senefiance ${ }^{\circ} 47$, Aix-en-Provence, Publications de l’Université de Provence, 2001, p. 382-394.

12 Dans l'illustration du mois de février des Très riches heures du duc de Berry (cf. illustration 3, p. 31), deux des personnages se réchauffant près de la cheminée ont relevé leurs vêtements. L'étrange exhibition de leurs parties intimes peut être lue comme une façon de figurer le dénuement des paysans. Cf. Jean-Luc DuFresne, "L'Obscène médiéval », in Sylvain FlOC'H (dir.), L'Obscène, Pau, Université de Pau et des pays de l'Adour, « Cahiers de l'Université », 4, 1983, p. 83-94 (p. 89).
} 
les milieux monastiques puisqu'il faut éviter de voir la nudité de ses coreligionnaires autant que la sienne propre ${ }^{13}$.

Néanmoins la pratique du bain, héritage antique, est courante et l'on $\mathrm{s}^{\prime} y$ rend en groupe ${ }^{14}$. Si les conciles successifs ont réussi à faire interdire l'usage des bains mixtes, le retour des croisés d'Orient, au XII ${ }^{\mathrm{e}}$ siècle, en ramène la pratique ${ }^{15}$. Très vite, à côté d'établissements d'utilité publique, ouvrent des étuves mixtes, dont la débauche offusque très tôt les autorités : on réglemente leur fréquentation et on instaure des jours pour chacun des deux sexes ${ }^{16}$. Aucune ordonnance n'y impose le port d'un vêtement mais les actes scandaleux sont interdits. La nudité est donc tolérée, mais ne doit pas donner lieu à des gestes licencieux.

Dans une telle perspective, toute représentation érotisante du corps pose problème. Pourtant la nudité est évoquée dans certaines œuvres littéraires ou dans les vies de saints, mise en scène dans des miniatures réalistes, les marges des manuscrits et même dans les lieux saints... Mais très souvent, sous l'influence de l'idéologie chrétienne, les auteurs et les artistes prennent leurs précautions pour ne pas donner l'impression de chercher à exciter le plaisir, hautement condamnable, et rappeler au contraire la puissance de la chair et l'état de pécheur de l'homme.

Si à la fin du Moyen Âge se fait jour une revalorisation du corps, il faut attendre la Renaissance pour revoir des images de nus ouvertement

\footnotetext{
${ }^{13}$ Cf. Jean-Claude Bologne, Histoire de la pudeur, Paris, Hachette, « Pluriel », 1987 ( $1^{\text {ère }}$ éd. Orlan, 1986), p. 112.

${ }^{14}$ Cf. Le Bain. Espace et pratiques médiévales, Didier BoISSEUIL (dir.), Paris, 2003 et Laver, monder, blanchir. Discours et usages de la toilette dans l'Occident médiéval, Sophie AlBERT (dir.), Paris, PUPS, 2006.

15 On en compte vingt-six à Paris en 1292. Cf. Jean-Claude Bologne, Histoire de la pudeur, op. cit., p. 30.

${ }^{16}$ Notons qu'une des peines que l'on a pu imposer aux contrevenants était de rentrer chez eux nus ou en chemise, ce qui, comme nous l'avons vu, revient au même. Cf. JeanClaude Bologne, Histoire de la pudeur, op. cit., p. 31.
} 
transgressives : elles choquent et sont donc condamnées ${ }^{17}$. En effet, le retour à l'Antique permet de se libérer de toute religiosité et de se réapproprier un corps représenté pour lui-même.

\section{Des pratiques sexuelles contrôlées}

L'essentiel du discours sur la sexualité est tenu par les clercs ${ }^{18}$. Très contrôlées, les pratiques sexuelles médiévales sont surtout connues par des témoignages juridiques ou des traités médicaux ${ }^{19}$. C'est la morale chrétienne qui détermine la régulation de la sexualité mais, depuis Paul Veyne, nous savons que ces principes sont mis en place dès le haut Empire romain $^{20}$ : le passage d'une «aristocratie concurrentielle » à une « aristocratie de service » va faire de l'amour et de la fidélité conjugale le modèle dominant, modèle que s'appropriera le christianisme naissant, lui donnant, sous l'influence de saint Jérôme et saint Augustin, une impulsion décisive. Mais l'héritage antique est plus vaste encore puisqu'une grande partie du savoir lié au sexe provient des traités antiques. On considère notamment que «la santé mentale est inversement proportionnelle à la vigueur génitale $»^{21}$. S'ajoute à cette défiance l'idée que le corps sexué est directement lié à la chute et donc irrémédiablement entaché, intrinsèquement mauvais.

\footnotetext{
${ }^{17}$ L'un des exemples les plus célèbres en est certainement le Jugement dernier de la Chapelle Sixtine dont les sexes furent pudiquement cachés et que la récente restauration n'a d'ailleurs pas dévoilés.

${ }^{18}$ Cf. Jacques VoISENET, «Mariage et interdits sexuels au Moyen Âge $\left(\mathrm{V}^{\mathrm{e}}-\mathrm{XII}{ }^{\mathrm{e}}\right.$ siècle) », in Sex, Love and Marriage in Medieval Literature and Reality, Thematische Beiträge im Rahmen des $31^{\text {th }}$ International Congress on Medieval Studies an der Western Michigan University (Kalamazoo - USA) 8-12 mai 1996, Greifswald, Reineke, «Wodan », 69, 1996, p. 53-72.

${ }^{19}$ Cf. Danielle JACQUART et Claude ThOMASSET, Sexualité et savoir médical au Moyen Âge, Paris, PUF, « Les chemins de l'Histoire », 8, 1985.

${ }^{20}$ Cf. Paul Veyne, «La famille et l'amour sous le Haut-Empire romain », art. cit., p. 40-41.

${ }^{21}$ Jacques Rossiaud, entrée « sexualité », in Jacques LE GoFF et Jean-Claude ScHMITT (dir.), Dictionnaire raisonné de l'Occident médiéval, Paris, Fayard, 1999, p. 1069.
} 
Il est donc nécessaire, pour le pouvoir clérical, d'encadrer très fortement la sexualité. C'est le rôle qui est dévolu au mariage qui définit une « copulation juste $»^{22}$. Hors du mariage le sexe est condamné et, à l'intérieur même de ce cadre, les jours où le coït est autorisé sont très limités, du moins en théorie ${ }^{23}$. La mise en place de pénitentiels permet alors d'associer une peine à chaque péché de chair. Il semble que ces prescriptions aient trouvé un écho dans la culture du temps, ce qui aurait entraîné une convergence de ces prescriptions avec les pratiques ${ }^{24}$.

Les femmes sont les premières victimes de ces règles puisqu'elles sont du côté de la chair. Leur infériorité sociale trouve un écho dans les interdits imposés à leur sexualité par la religion : l'equus eroticus où la femme domine l'homme est évidemment proscrit, la procréation pendant les menstruations des femmes est supposée donner naissance aux lépreux et, plus largement, toute procréation relevant d'un interdit est réputée engendrer malades et monstres ${ }^{25}$.

\section{Les excréments : souillure et rebuts du corps}

Les fonctions excrémentielles du corps sont moins suspectes que ses fonctions sexuelles. Dans l'univers médiéval, les matières fécales, sans être valorisées, ne sont pas encore seulement sales. Il ne faut pas oublier qu'elles ont de nombreux aspects utiles : le fumier sert à engraisser le sol,

\footnotetext{
${ }^{22}$ Jacques LE Goff et Nicolas TruOng, Une Histoire du corps au Moyen Âge, op. cit., p. 45.

${ }^{23}$ On sait que les prescriptions ne sont pas toutes les mêmes selon les périodes. Les plus répressives n'autorisent le coït que 91 jours par an (VIII ${ }^{\mathrm{e}}$ siècle); ces contrôles très stricts vont néanmoins s'assouplir au fil des siècles. Cf. Jean-Louis FLANDRIN, Un Temps pour embrasser. Aux origines de la morale sexuelle occidentale ( $V I^{e}-X I^{e}$ siècles), Paris, Seuil, 1983 et Jacques LE GofF et Nicolas TrUONG, Une Histoire du corps au Moyen Âge, op. cit., p. 43-46.

${ }^{24}$ C'est à partir de l'étude de la démographie qu'a pu être mis en évidence le respect des prescriptions cléricales. Cf. Jacques LE GOFF et Nicolas TRUONG, Une Histoire du corps au Moyen Âge, op. cit., p. 43-46.

${ }^{25}$ Cf. Jacques Rossiaud, entrée «sexualité », in Dictionnaire raisonné de l'Occident médiéval, op. cit., p. 1071.
} 
les urines sont utilisées à des fins médicales, certaines déjections entrent dans la composition de matériaux de construction... Leur utilisation fait partie du quotidien. Pour le clergé, en revanche, elles renvoient à « la vile ignominie de l'existence humaine $»^{26}$. L'emploi de termes renvoyant aux excréments dans la formation des injures montre assez le peu d'estime pour cette matière.

Les représentations scatologiques ne manquent pas, dans la ville, aussi bien que dans les livres ${ }^{27}$. Les marges des manuscrits autant que les fabliaux présentent des jeunes gens offrant leurs excréments à leur dame et les scènes de coprophagie y sont nombreuses. Un système d'inversion se fait jour dans l'utilisation de cette vile matière qui permet de renverser les stéréotypes chevaleresques et courtois ou de faire se prosterner une sainte devant un anus ${ }^{28}$. Mais il ne faut pas oublier une autre symbolique importante, l'association de la merde et de l'argent. Le flux produit est souvent offert comme de l'argent et la matière morte est ainsi sans cesse remise en circulation, ce que certains exégètes des fabliaux proposent de lire comme une image de la matière littéraire sans cesse réutilisée ${ }^{29}$.

Nous savons que la grivoiserie, la pornographie et la scatologie naissent de la transgression des limites juridiques et morales qui dictent ce qu'il est permis ou ce qu'il est décent de montrer et de dire. Même si la longue période du Moyen Âge connaît nécessairement des évolutions, nous pouvons retenir que la sexualité est de façon générale considérée comme un mal nécessaire et que l'évocation quelle qu'elle soit du bas-corporel est sentie comme obscène.

\footnotetext{
${ }^{26}$ La remarque est attribuée au pape Innocent III, cité par Michael CAMILLE, Images dans les marges. Aux limites de l'art médiéval, Paris, Gallimard, "Le Temps des images », 1997 ( $1^{\text {ère }}$ éd. américaine 1992), p. 154.

${ }^{27}$ Cf. illustrations 1 et 2 , p. 31 .

${ }^{28}$ Cf. image marginale du manuscrit du Roman d'Alexandre, Oxford, Bodleian Library, Bodl. 264, $\mathrm{f}^{\circ} 56 \mathrm{r}^{\circ}$.

${ }^{29}$ Cf. Michael CAMILLE, Images dans les marges, op. cit., p. 158.
} 


\section{Les modes de transgression : dire et montrer}

S'il est difficile de connaître avec certitude la réalité des pratiques attachées à la nudité et à la sexualité dans l'intimité des foyers médiévaux, nous constatons en revanche que leur évocation et leur représentation sont volontiers censurées. Il importe maintenant de nous pencher sur les modes de transgression mis en œuvre pour contourner ces interdits.

\section{Le mot et la chose ${ }^{30}$}

Les représentations iconographiques mettaient en scène des comportements souvent assez lestes pendant l'Antiquité, mais Cicéron, dans son traité De Officiis, recommande déjà une certaine pudeur dans l'écriture :

Hanc naturae tam diligente fabricam imitata est hominum uerecundia. Quae enim natura occultauit, eadem omnes qui sana mente sunt, remouent ab oculis ipsique necessitati dant operam ut quam occultissime pareant; quarumque partium corporis usus sunt necessarii, eas neque partes neque earum usus suis nominibus appellant; quodque facere non turpe est, modo occulte, id dicere obscenum est. Itaque nec actio rerum illarum aperta petulantia uacat nec orationis obscenitas. $^{31}$

Le christianisme a accentué cette exigence de bienséance et saint Jérôme lui-même, citant Job, invite à la plus grande retenue lorsqu'il s'agit de nommer les genitalia:

\footnotetext{
${ }^{30} \mathrm{Ce}$ titre est emprunté au célèbre poème galant de Gabriel-Charles de Lattaignant.

31 «La pudeur des hommes a imité cet art si attentif de la nature. Ce que la nature en effet a dissimulé, c'est cela même que tous ceux qui sont sains d'esprit, éloignent des regards, et ils s'efforcent, aux besoins mêmes de la nature, d'obéir le plus secrètement possible. Quant à ces parties du corps dont l'usage est indispensable, ils n'appellent par leurs noms, ni ces parties ni leur usage, et il est indécent de dire ce qu'il n'est point honteux de faire, du moins en secret. C'est pourquoi l'acte de ces fonctions, en public, ne manque pas d'impudence, et l'indécence ne manque pas non plus à en parler. », CicÉron, Les Devoirs, Maurice Testard (éd.), Paris, Les Belles Lettres, 1974, Livre I, XXXV, p. 170-171.
} 
Iob deo carus et testimonio ipsisus inmaculatus et simplex, audi quid de diabolo suspicetur : " uirtus eius in lumbis et potestas eius in umbilico ». Honeste uiri mulierisque genitalia inmutatis sunt appellata nominibus. ${ }^{32}$

Cette tendance s'affirmera encore au Moyen Âge. Même employés sans arrière-pensée, les mots renvoyant à la sexualité demeurent suspects, au point que dans les textes scientifiques un vocabulaire médical se développe, distinct du langage commun, comme en témoigne cet exposé extrait du Placides et Timéo :

Il couvient que li malles ait ces deux genitaires que je vous ai dit qui sont apelees «testiculi » en latin et c'est a dire petis tesmoings du sexe a l'omme pour savoir se il est malles ou fumelle. Et si couvient que li homs ait une vergue [...] la quelle vergue est apelee «preape » et en commune langue « vit ».33

Pour écarter tout soupçon de concupiscence, les scientifiques mettent au point un langage qui leur est propre et leur permet d'évoquer les fonctions sexuelles ou excrémentielles du corps sans détours rhétoriques.

La question de la nomination des réalités sexuelles en littérature témoigne de ce souci de bienséance. Les auteurs médiévaux de la littérature dite «noble » répugnent en effet à employer des mots considérés comme grossiers parce que trop explicites. Dans les textes courtois où l'amour tient une place centrale, plusieurs stratégies sont mises en œuvre pour évoquer la rencontre charnelle avec une certaine pudeur. L'euphémisme, parce qu'il atténue toute idée qui pourrait heurter les sensibilités, est souvent employé : par exemple l'acte sexuel est volontiers désigné par les mots « fag » dans la

\footnotetext{
32 «Job, cher à Dieu et d'après son propre témoignage, immaculé et simple, écoute de quoi il soupçonne le diable: "Sa force est dans les reins et sa puissance dans le nombril"; c'est une manière honnête de désigner par des euphémismes les parties génitales de l'homme et de la femme », SAINT JÉRÔME, Lettres, t. I, Jérôme LABOURT (éd et trad.), Paris, Les Belles Lettres, 1982, Epist. 22. 11.2, p. 120-121.

33 Placides et Timéo ou Li secrés as philosophes, Claude THOMASSET (éd.), Paris/Genève, Droz, 1980, p. 100-101.
} 
poésie occitane, «deduit» ou «delit» dans les récits en langue d'oïl, tandis que pour éviter de mentionner les parties génitales, on utilisera la métonymique «cuisse » ou le mystérieux «surplus». Ailleurs c'est la litote qui, disant le moins pour suggérer le plus, rend compte du plaisir pris par les amants. Ainsi dans Le Donnei des amants, lorsque Tristan et Yseut peuvent enfin se retrouver seuls, ils «ovrent assez e poi parolent $»^{34}$. L'ellipse est également un procédé littéraire fréquent et nous pouvons remarquer que nombre de descriptions s'arrêtent fort opportunément à la taille. La métaphore est en définitive moins courante et apparaît essentiellement dans les textes comiques.

Ce problème de la nomination est au cœur de certaines intrigues, comme dans le Lay del Lecheor par exemple ou, de façon plus évidente, dans plusieurs fabliaux. Le Lay del Lecheor ${ }^{35}$ raconte comment, lors d'une fête, une assemblée courtoise écoute des chevaliers relater leurs exploits ; un lai sera composé pour conserver le souvenir de l'aventure la plus audacieuse. Une femme prend la parole pour faire remarquer qu'on ne rend jamais hommage à ce qui motive tous ces exploits : le sexe de la femme ! La révélation de l'objet digne de tous les honneurs est retardée et l'originalité du texte naît du contraste entre le contexte courtois et la verdeur des propos de la dame. Mais, à la fin du texte, le narrateur renonce à citer le véritable titre du lai :

D'icest lai dient li plusor que c'est le lai du Lechëor ; ne voil pas dire le droit non c'on nu me trot a mesprison. ${ }^{36}$

\footnotetext{
${ }^{34}$ Le Donnei des amants, in Tristan et Iseut. Les poèmes français, la saga norroise, Daniel Lacroix et Philippe WALTER (éd.), Paris, LGF, «Le Livre de Poche. Lettres Gothiques », 1989, p. 324, v. 200.

${ }^{35}$ Lay del Lecheor, in Lais féériques des XII et XIII ${ }^{e}$ siècles, Alexandre MichA (éd.), Paris, Garnier-Flammarion, 1992, p. 333-341.

${ }^{36}$ Ibid., p. 341, v. 117-120.
} 
Contrairement au lai, le fabliau, qui passe pour être le genre médiéval le plus grivois, n'hésite pas à désigner les réalités sexuelles soit par des mots crus $^{37}$, soit par des expressions figurées mais néanmoins transparentes. Le comique revendiqué de ces textes autorise le dépassement du tabou, mais en atténue passablement l'aspect transgressif ${ }^{38}$. Certains auteurs de fabliaux se jouent cependant fort habilement de l'interdit en mettant en scène à la fois l'acceptation des pratiques sexuelles et le refus de leur désignation. Dans La Damoisele qui ne pooit oür parler de foutre $e^{39}$, l'héroïne ne supporte pas que l'on parle de sexualité devant elle, mais accepte facilement une relation charnelle dont elle détaillera oralement chaque étape en filant une métaphore animale.

En dehors de tout contexte comique, Jean de Meun est un des premiers à revendiquer l'emploi de termes propres pour désigner les organes sexuels. Dans Le Roman de la rose, lors d'une discussion entre l'amant et Raison, cette dernière nomme les couilles et le vit et explique son audace :

Se je, quant mis les nons aus choses que si reprendre et blasmer oses, coilles reliques apelasse et reliques coilles clamasse, tu, qui si m'en morz et depiques, me redeïsses de reliques que ce fust lez moz et vilains. Coilles est biaus nons et si l'ains,

\footnotetext{
37 Notons cependant que le vocabulaire est peu étendu : «vit», «con», «cul» et « foutre » sont de loin les termes les plus fréquents. Cf. Jean SCHEIDEGGER, « Gros mots gros rires? Le comique sémiologique de quelques fabliaux scabreux », in Thérèse Bouché et Hélène CHARPEnTIER (dir.), Le Rire au Moyen Âge dans la littérature et dans les arts, Bordeaux, Presses Universitaires de Bordeaux, 1990, p. 309-320.

38 Sur ce point, voir Bernard RIBEMONT, Sexe et amour au Moyen Âge, Paris, Klincksieck, « 50 Questions », 2007, p. 109-112 et Jean-Claude AUBAILLY, « Le fabliau et les sources inconscientes du rire médiéval », Cahiers de Civilisation Médiévale, XXX (1987), p. 105-117.

${ }^{39}$ La Damoisele qui ne pooit oür parler de foutre, in Fabliaux érotiques. Textes de jongleurs des XII et XIII $I^{e}$ siècles, Luciano ROSSI et Richard STRAUB (éd.), Paris, LGF, «Le Livre de Poche. Lettres gothiques », 1992, p. 89-105.
} 
si sunt par foi coillon et vit, onc nus plus biaus guieres ne vit. ${ }^{40}$

Elle finira par concéder que l'usage, dont elle souligne l'arbitraire, veut que l'on évite d'employer les mots propres. Cependant il résulte de ce dialogue que l'obscénité n'est ni dans l'objet, ni dans le mot qui le désigne, mais dans l'esprit de celui qui n'ose pas nommer ce qu'il désire. Cette position nouvelle ne manquera pas d'entraîner une vive polémique ${ }^{41}$.

Dans le domaine de l'image, nous retrouvons la même aversion à voir figurer explicitement la sexualité. Certes, la représentation de la nudité est relativement fréquente lorsque le sujet religieux ou mythologique la légitime, encore qu'un voile ou une main vient souvent fort à propos couvrir les parties les plus intimes. Certains de ces nus ont toutefois paru trop évocateurs aux yeux des hommes du Moyen Âge qui ont préféré en effacer les zones les plus sensuelles sur des enluminures ${ }^{42}$. Plus que la nudité qui peut renvoyer à des notions positives comme l'innocence, la

40 Guilaume de Lorris et Jean de Meun, Le Roman de la Rose, Félix LeCOy (éd.), Paris, Champion, 1973, v. 7079-7088.

${ }^{41}$ Pour approfondir ce point, on lira avec profit Virginie GREEN, «Le débat sur le Roman de la Rose comme document d'histoire littéraire et morale », in Bruno MÉNIEL et Bernard RiBÉmont (dir.), La Figure de Jules César au Moyen Âge et à la Renaissance, II, Cahiers de recherches médiévales et humanistes, 13 (2006), p. 297-311; Yves FerRoul, «Réalités sexuelles et fiction romanesque », in Danielle BUSCHINGER (dir.), Les «Realia » dans la littérature de fiction au Moyen Âge, Amiens, Université de Picardie-Jules Verne, Presses du Centre d'Études Médiévales, 2000, p. 40-49; Thérèse BOUCHE, «L'obscène et le sacré ou l'utilisation paradoxale du rire dans le Roman de la Rose de Jean de Meun», in Le Rire au Moyen Âge dans la littérature et dans les arts, op. cit., p. 83-95 ; Daniel POIRION, «Les mots et les choses selon Jean de Meun », in L'Information littéraire, XXVI (1974), p. 7-11.

${ }^{42}$ Cf. illustration 4, p. 32. Cette belle représentation de Bethsabée au bain a semblé trop indécente à celui qui a gratté le corps de la jeune femme. Il est vrai que les cheveux dénoués, les paupières mi-closes, les joues rougissantes et les courbes généreuses sont de puissants indices érotiques que le léger voile couvrant le pubis ne suffit pas à contrebalancer. Pour une analyse des «raisons de détruire une image », voir Gil BARTHOLEYNS, Pierre-Olivier DitTMAR et Vincent JOLIVET, Image et transgression au Moyen Âge, Paris, PUF, «Lignes d'art », 2008, en particulier le chapitre V « Montages transgressifs », p. 127-150, et Gil BARThOLEYns, Pierre-Olivier DitTMAR et Vincent JOLIVET, «Des raisons de détruire une image», Images Re-vues, 2 (2006), http://imagesrevues.revues.org/248. 
sexualité est difficile à figurer sans risquer d'éveiller le désir. Le parti pris est généralement celui de la symbolisation, comme sur cette valve de miroir en ivoire où la couronne et les plis de la robe évoquent le désir féminin, tandis qu'un faucon et surtout un mas dressé entre des rideaux entrouverts ne laissent pas de doute sur les intentions de l'amant ${ }^{43}$.

Nous constatons finalement que le tabou concerne moins l'acte sexuel que son évocation. En général les artistes respectent l'interdit dont ils jouent: la contrainte se révèle pour eux une fructueuse source d'inspiration.

\section{La marge}

De façon générale l'exhibition du bas corporel semble confinée dans un espace marginal. Dans la ville, l'exposition de la nudité est concentrée dans des lieux clos, inaccessibles au simple regard, comme les latrines ou les étuves. L'obscénité sociale, pourrait-on dire, la plus évidente, à savoir la prostitution, est rejetée en périphérie des villes. Il en est de même pour les œuvres d'art: les représentations explicites de la sexualité ou de la scatologie sont, le plus souvent, réservées à des espaces marginaux.

À partir du XIII ${ }^{\mathrm{e}}$ siècle, les marges des manuscrits se couvrent de dessins parfois très étranges. Les fonctions de ces marginalia sont multiples. Elles permettent, entre autres, d'identifier le possesseur du manuscrit et de se repérer dans le livre; elles peuvent illustrer ou commenter le contenu du texte qu'elles accompagnent ou au contraire servir de contrepoint. Mais leur interprétation reste bien souvent problématique $^{44}$. Si Michael Camille voit dans les marges un discours

\footnotetext{
${ }^{43}$ Cf. illustration 5, p. 32. Pour une analyse de cette image, voir Michael CAMILLE, The Medieval Art of Love, Londres, Laurence King, 1998, p. 124.

${ }^{44}$ Cf. Jean-Claude SchMITT, «L'univers des marges », in Jacques DALARUN (dir.), Le Moyen Âge en lumière. Manuscrits enluminés des bibliothèques de France, Paris, Fayard, 2002, p. 329-361 et 389 ; Jean WIRTH, Les Marges à drôleries des manuscrits gothiques (1250-1350), Genève, Droz, 2008.
} 
contredisant l'ordre établi, de façon générale il faut toujours les lire dans la tension qu'elles créent avec le texte et avec l'ensemble du programme iconographique du manuscrit; les marges offrent souvent, sur le mode de l'humour, une relecture du texte proposé dans la page, qu'elles le glosent ou qu'elles en livrent une version plus courtoise ou galante ${ }^{45}$.

Les scènes à caractère scatologique ou sexuel sont nombreuses. Souvent figurées de façon allusive ou métaphorique, il arrive qu'elles soient franchement obscènes, comme sur ce manuscrit du Roman de la Rose illustré par Jeanne de Montbaston où une nonne cueille les fruits d'un arbre à vits, avant de se faire trousser par un moine ${ }^{46}$ ! L'interprétation de ces scènes peu équivoques impose de les mettre en relation avec les différents dessins du manuscrit qui critiquent le clergé en le ridiculisant. Elles illustrent aussi directement le texte :

Par cloistres et par abbaïes sunt toutes contre lui [Chasteté] jurees ; ja si ne seront enmurees que Chastaé si fort ne heent que toutes a honir la beent. ${ }^{47}$

La conception de la sexualité qui émane du manuscrit est problématique puisque ces images illustrent un texte qui dédramatise une sexualité présentée comme naturelle tout en participant de la critique du clergé dont elles raillent la concupiscence.

En ce qui concerne la littérature, la présence de mots crus pour évoquer les fonctions sexuelles et excrémentielles est attestée essentiellement dans trois types de textes bien précis. Le premier correspond aux textes comiques. Comme nous l'avons mentionné, leur pouvoir de transgression demeure limité, car tous ces termes ne sont

45 Cf. Markus MülleR, «Fonctions du profane et du "ridiculum" dans l'enluminure médiévale », Histoire de l'art, 29/30 (mai 1995), p. 23-31.

${ }^{46} \mathrm{Cf}$. illustrations 6 et 7 , p. 33 .

47 Guillaume De LoRris et JeAn De Meun, Le Roman de la Rose, éd. cit., v. 8990-8994. 
employés que «pour rire ». À l'opposé, des textes édifiants peuvent avoir recours au même vocabulaire. L'obscénité se veut alors réaliste et grave pour mieux rendre la grossièreté du vice qu'elle condamne, à l'instar de cette réflexion d'Odilon, abbé de Cluny, au $\mathrm{X}^{\mathrm{e}}$ siècle :

La beauté physique ne va pas au-delà de la peau. Si les hommes voyaient ce qui est sous la peau, la vue des femmes leur soulèverait le cœur. Quand nous ne pouvons toucher du bout du doigt un crachat ou de la crotte, comment pouvonsnous désirer embrasser ce sac de fiente ? $^{48}$

Enfin, la scatologie et la sexualité sont bien présentes dans ce que Pierre Bec nomme «le contre-texte » et qu'il définit comme « un texte minoritaire et marginalisé, une sorte d'infra littérature [dont la] réception et [1']impact sont liés aux modalités du code textuel majoritaire ${ }^{49}$. Le contre-texte utilise donc les thèmes sexuels et scatologiques pour mieux pervertir un code littéraire normé. La poésie goliardique en est un bon exemple.

Ainsi, la présence d'un bas corporel dénigré hésite le plus souvent entre deux extrêmes: la métaphore, dont le champ extrêmement large couvre l'érotisme le plus délicat jusqu'à la grivoiserie la plus grasse, et l'expression libre et crue, mais réservée à un espace d'expression marginal.

\section{L'exemple des images sexuelles dans les lieux de culte}

Afin d'illustrer la difficulté à interpréter les représentations médiévales du bas-corporel, nous nous proposons de développer, pour terminer, une analyse des images sexuelles dans les lieux de cultes médiévaux, à partir de deux types d'exemples: les obscenas, statues

\footnotetext{
${ }^{48}$ Cité par Jean Delumeau, La Peur en Occident, Paris, Fayard, 1978, p. 409.

${ }^{49}$ Pierre BEC, Burlesque et obscénité chez les troubadours : le contre-texte au Moyen Âge, Paris, Stock, 1984, « Moyen Âge », p. 11-13.
} 
d'exhibition et de coït que l'on trouve dans les églises, et les enseignes de pèlerinage dites profanes ${ }^{50}$.

\section{Perplexité du regard moderne sur ces images}

Aujourd'hui encore, le fidèle averti peut découvrir dans les églises des figures féminines exhibant leur vulve ou des figures masculines arborant un membre pour le moins vigoureux, parfois aussi un «chieur» ou un couple enlacé ${ }^{51}$. Quant aux archéologues, ils ont mis à jour un certain nombre d'enseignes de pèlerinage intrigantes : vulves pèlerines, verges sur pattes, moines soulevant leur robe ${ }^{52} \ldots$ Faites d'un même alliage que les enseignes dévotes, vendues par les mêmes artisans, découvertes au même endroit, ces petites broches que le pèlerin portait sur son chapeau ne manquent pas d'intriguer. Par leur impudeur et par leur localisation, ces images explicites ont plongé l'observateur moderne dans une certaine perplexité. On y a d'abord vu une représentation du mal, de la luxure, une forme de contre-modèle à rejeter. L'emplacement des statues les plus obscènes, généralement situées sur les façades des églises, laissées à l'extérieur donc, peut être un argument allant dans ce sens. L'obscénité de ces représentations serait justifiée par leur rôle dans l'édification du fidèle. De fait la petite sculpture d'un personnage nu se masturbant dans une frise de la cathédrale de Chartres permet d'identifier, parmi d'autres personnages bibliques, la statue d'Onan qu'elle accompagne. Toutefois ces interprétations demeurent insatisfaisantes pour de nombreuses sculptures et

\footnotetext{
${ }^{50}$ Ce développement doit beaucoup à deux ouvrages auxquels nous renvoyons : Gil BARTHOLEYNS, Pierre-Olivier DITTMAR et Vincent JOLIVET, Image et transgression au Moyen Âge, op. cit., (notamment le chapitre IV «L'épreuve du temps », p. 99-125) et Denis BRUNA, Enseignes de plomb et autres menues chosettes du Moyen Âge, Paris, Le Léopard d'Or, 2006, chap. XI « Des enseignes obscènes, indignes et nues », p. 207-230. ${ }^{51}$ Cf. illustrations 8 et 9 , p. 34. Pour une idée plus complète de cette production, on peut se référer aux sites d'amateurs éclairés http://web.me.com/joel.jalladeau/obscenite/ et http://www.beyond-the-pale.org.uk/satan1.htm.

${ }^{52}$ Cf. illustrations 10 et 11, p. 34.
} 
pour l'ensemble des enseignes. L'anticléricalisme du début du $\mathrm{XX}^{\mathrm{e}}$ siècle a voulu y voir une preuve de la lubricité des prêtres ou du double discours de l'Église qui condamne une sexualité autrefois acceptée dont témoignent ces représentations $^{53}$. Mais trop dépendante du contexte de son élaboration, cette hypothèse est largement abandonnée.

\section{Fonctions apotropaïque et votive}

Plus récemment, on a proposé de voir dans l'utilisation de ces images sexuelles une fonction apotropaïque, c'est-à-dire protectrice, héritée de cultes antiques $^{54}$. Ainsi les représentations de phallus de taille démesurée ou munis de pattes ou de clochettes rappellent le culte de Priape. L'exhibition des vulves, parfois sous la forme de «sheela-na-gig», personnages féminins grotesques montrant leurs parties génitales, surtout présentes en Irlande et au Royaume Uni, porterait la trace du paganisme celte $^{55}$. On a pu voir aussi, mais sans le prouver, un lien avec la déesse grecque Baubo qui mit fin à la tristesse de Déméter en lui montrant son anatomi $^{56}$. Ainsi sculptées sur les façades des églises, mais aussi parfois des châteaux, ou portées sur les vêtements des pèlerins, ces images sexuelles éloigneraient les influences maléfiques ${ }^{57}$. Cette fonction protectrice des images sexuelles existe encore dans d'autres cultures, notamment en Asie. Deux interprétations apparemment contradictoires

\footnotetext{
${ }^{53}$ Cf. Jean-Luc DuFRESNE, «L'Obscène médiéval », art. cit., p. 83-94.

${ }^{54}$ Cf. Alessia TRIVELlONE, «Têtes, lions et attributs sexuels : survivances et évolutions de l'usage apotropaïque des images de l'Antiquité au Moyen Âge », in Actualité de l'art antique dans l'art roman, Les Cahiers de Saint-Michel de Cuxa, XXXIX (2008), p. 209-221.

${ }^{55}$ Sur l'exhibition du sexe féminin, voir Patrizia CASTELLI, « Il doppio significato. L'ostensione della vulva nel medioevo », in S. BERTELLI et M. CENTANNI (dir.), Il gesto nel rito e nel ceremoniale dal monde antico ad oggi, Florence, Ponte alle Grazia, 1995, p. 199-223.

${ }_{56}$ Cf. Denis Bruna, Saints et diables au chapeau, Paris, Seuil, 2007, p. 155-161.

57 Cf. Gil BARtholeyns, Pierre-Olivier DitTMAR et Vincent Jolivet, Image et transgression ..., op. cit., p. 110-115.
} 
peuvent expliquer cette représentation positive du bas-corporel : soit ce qui donne la vie éloignerait la mort, soit le mal repousserait le mal ${ }^{58}$.

À cette fonction apotropaïque pourrait s'ajouter, pour les enseignes, une fonction votive. Nombre d'entre elles ont été retrouvées dans les fleuves. Elles portent parfois une croix sur leur revers ou ont été pliées selon un schéma particulier qui laisse supposer un geste rituel. Il s'agit alors probablement d'ex-voto portant l'image de l'organe souffrant pour lequel on entreprenait un pèlerinage $\mathrm{e}^{59}$.

\section{Superposition des lectures et naissance de la pornographie}

En définitive, toutes les interprétations que nous venons de citer ne s'excluent pas, mais plutôt se superposent, et cela dès le Moyen Âge. L'image d'un sexe était à la fois repoussante et bienveillante. Se poser la question du choix, en dehors d'un usage votif, d'une enseigne plutôt que d'une autre est intéressant. Le pèlerin qui préfère acheter une vulve en procession, plutôt qu'une tête de saint Jean Baptiste, n'est-il pas séduit par la grivoiserie de la scène, un peu comme le touriste en goguette s'achète un souvenir ou une carte postale de ce type dans une boutique de Montmartre? L'achat même de ces enseignes nous rappelle d'ailleurs la dimension vénale de la pornographie moderne que nous avons évoquée en introduction. Les enseignes représentent en effet un marché juteux qui oppose parfois les abbayes qui fabriquent ces objets aux artisans qui ont la même activité ${ }^{60}$.

À la fin du Moyen Âge, le regard porté sur ces images sexuelles change. Elles sont de plus en plus réduites à leur caractère sensuel et tombent dans le domaine exclusif du profane. Ainsi, à la fin du $\mathrm{XIV}^{\mathrm{e}}$ siècle,

\footnotetext{
${ }^{58}$ C'est encore le cas lorsque l'on dit «merde » aujourd'hui pour souhaiter bonne chance.

${ }^{59}$ Cf. Denis BrunA, Saints et diables au chapeau, op. cit., p. 149-164.

${ }^{60}$ Ibid., p. 45-56.
} 
Jean Gerson fustige l'indécence de certaines statues dans les églises. La naissance de la pornographie pourrait être envisagée comme une conséquence indirecte de cette crispation.

Même si, tout au long du Moyen Âge, la perception du bas corporel évolue, le discours clérical qui constitue la norme morale n'a de cesse de le dévaloriser. Les représentations d'activités sexuelles et excrémentielles sont toutefois nombreuses dans le quotidien des médiévaux et prennent tout leur sens par rapport à cette norme qu'elles entérinent, soit en lui servant de contre-modèle, soit en la reconnaissant comme modèle à transgresser.

Les mises en scènes sensuelles et scatologiques sont d'autant plus difficiles à interpréter aujourd'hui qu'elles étaient déjà équivoques au Moyen Âge, où elles oscillaient entre domaines religieux et profane, christianisme et paganisme, édification et divertissement...

À la fin de la période médiévale, le poids des interdits se fait plus lourd. La scatologie devient indissociable du rire. La représentation réaliste de la sexualité perd progressivement toute possible connotation positive lorsqu'elle n'est pas comique. Mais l'interdit plus fort que fait peser sur elle la morale fait naître de nouvelles émotions : défendue, elle n'en est que plus désirable. Son expression relève désormais de la grivoiserie ou de la pornographie. 


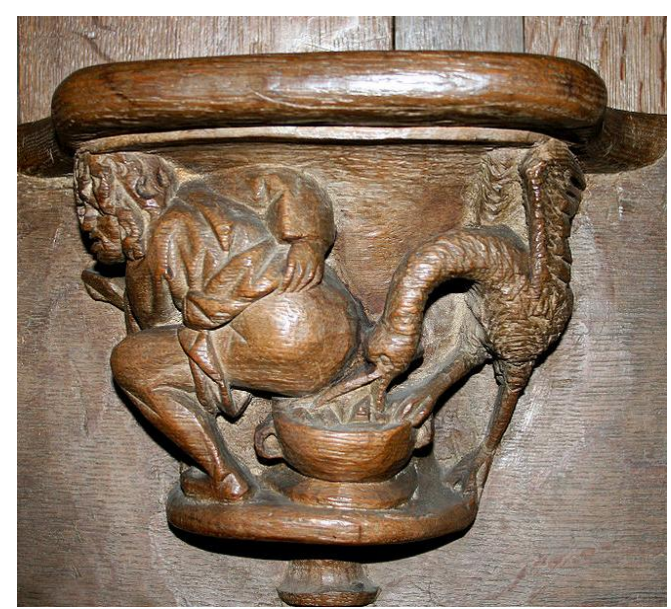

\section{Illustration 1}

Miséricorde de la basilique Saint-Materne, $\mathrm{XVI}^{\mathrm{e}}$ siècle, Walcourt, Belgique. Crédit photo : Jean-Pol Grandmont.

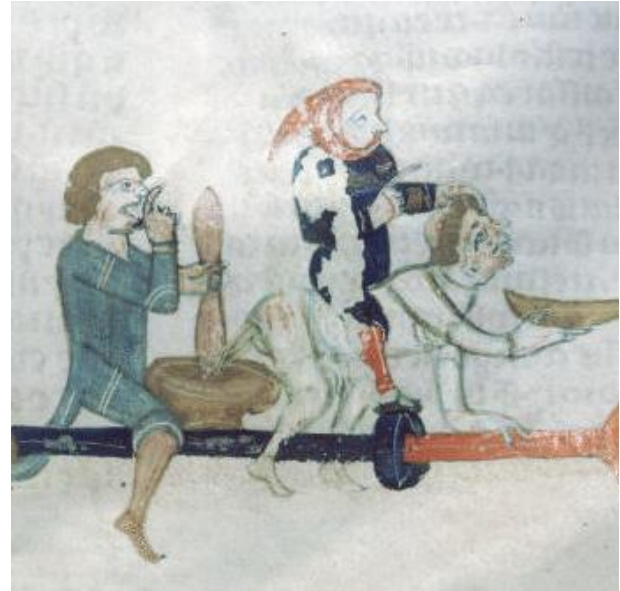

\section{Illustration 2}

«Scène scatologique », Arbor consanguinitatis et arbor affinitatis, XIII $^{\mathrm{e}}$ siècle, Tours, BM 0568, $\mathrm{f}^{\circ} 121$.

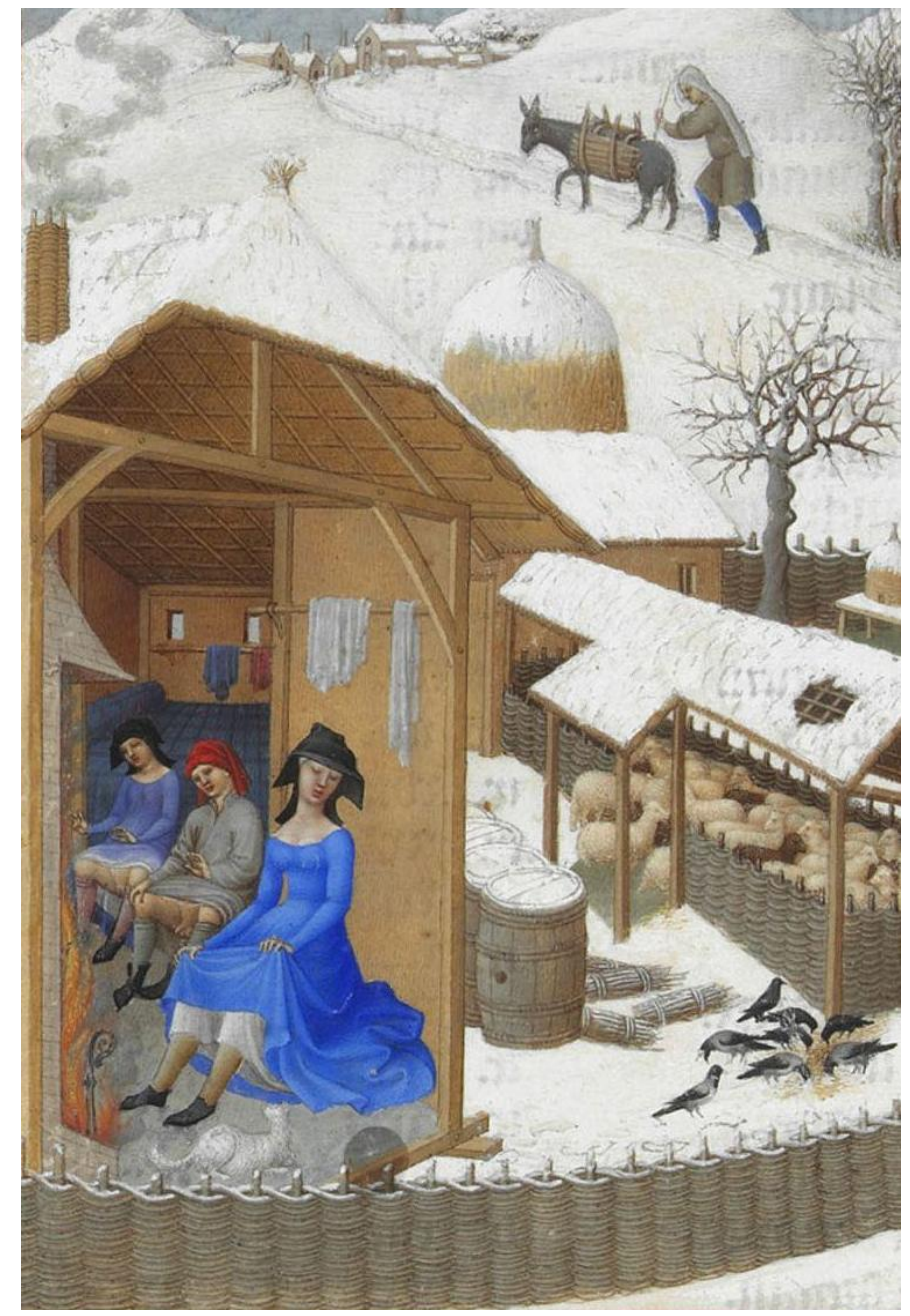

Illustration 3

«Février » (détail), Les Très riches heures du duc de Berry, XV siècle,

Chantilly, Musée Condé. 


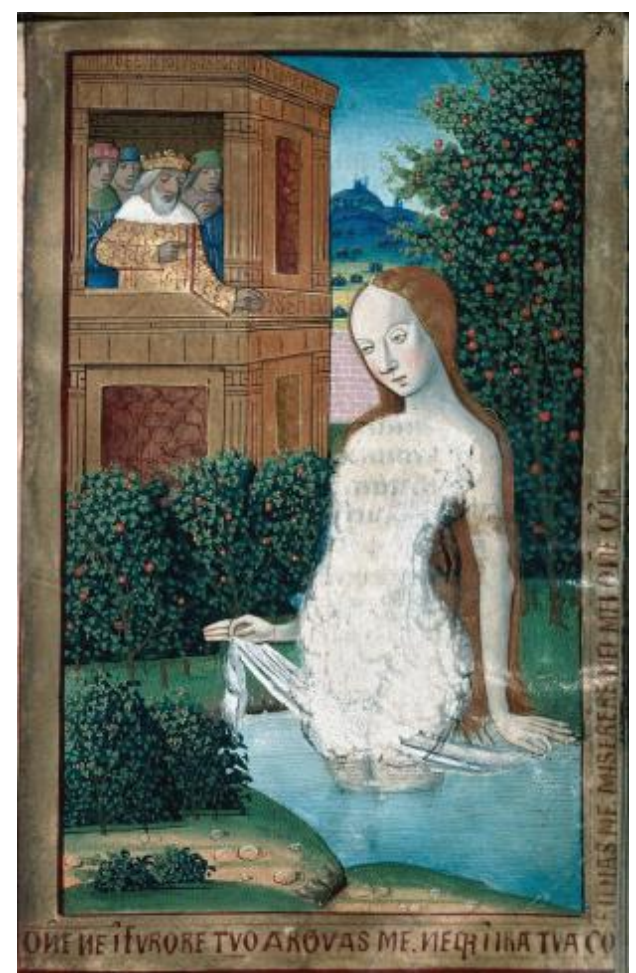

Illustration 4

« David et Bethsabée », Heures à l'usage de Sarum et de Poitiers, fin $\mathrm{XV}^{\mathrm{e}}$ siècle, Besançon, BM 0136, f 54.

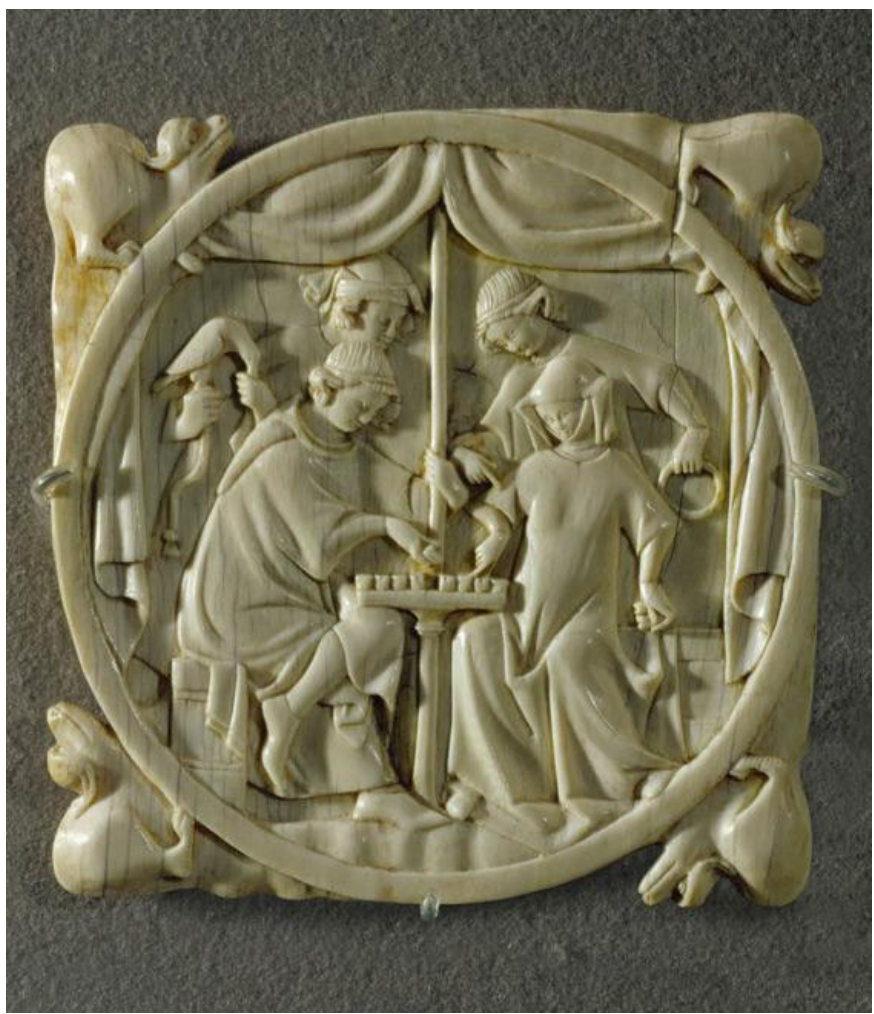

Illustration 5

«La partie d'échecs », valve de miroir, XIV ${ }^{\mathrm{e}}$ siècle, ivoire $(11 \times 5 \mathrm{~cm})$

Paris, Musée du Louvre, OA 117. 


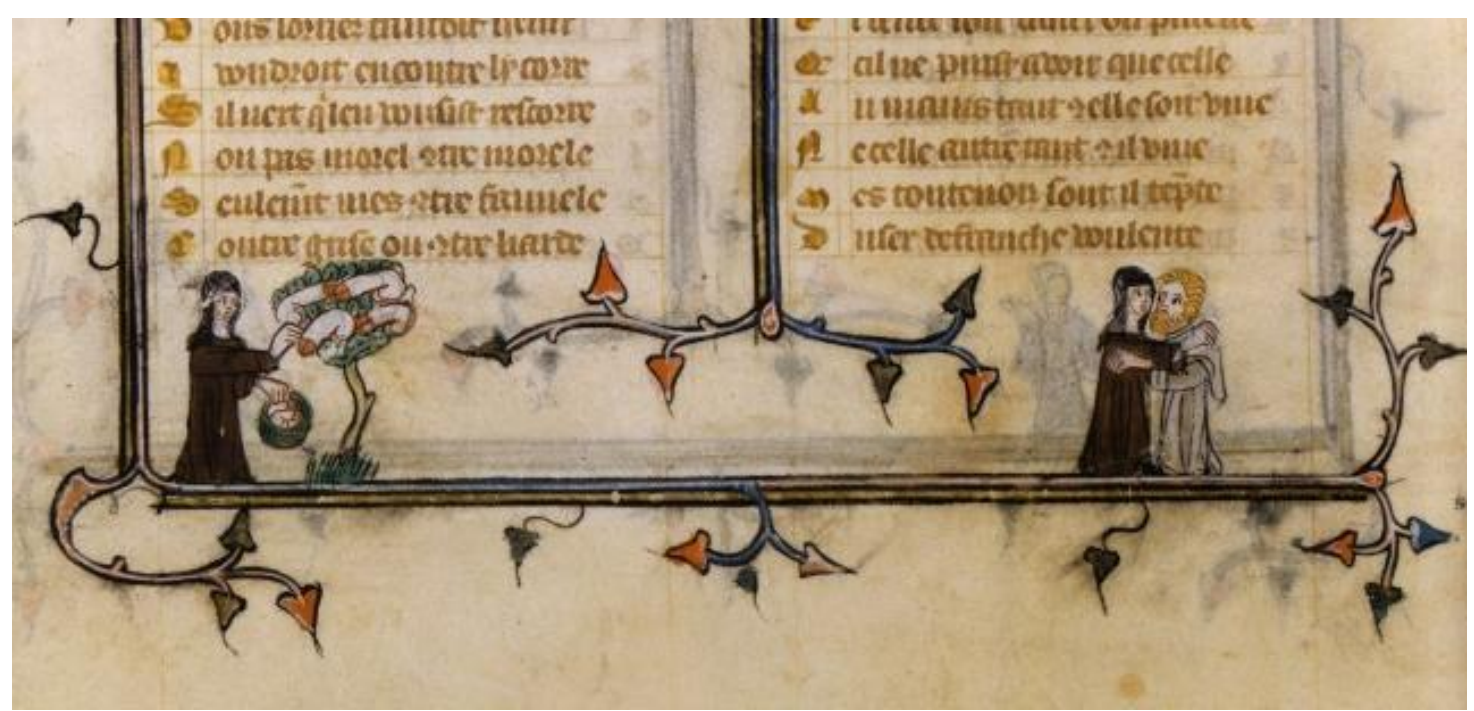

Illustration 6

Jeanne de Montbaston, « Nonette faisant la cueillette des vits », GuILlaume DE LORRIS et JEAN DE MEUn, Le Roman de la Rose, fin du XIV ${ }^{\mathrm{e}}$ siècle, Paris, BnF, f. fr. 25526, $\mathrm{f}^{\circ} 106 \mathrm{v}^{\circ}$.

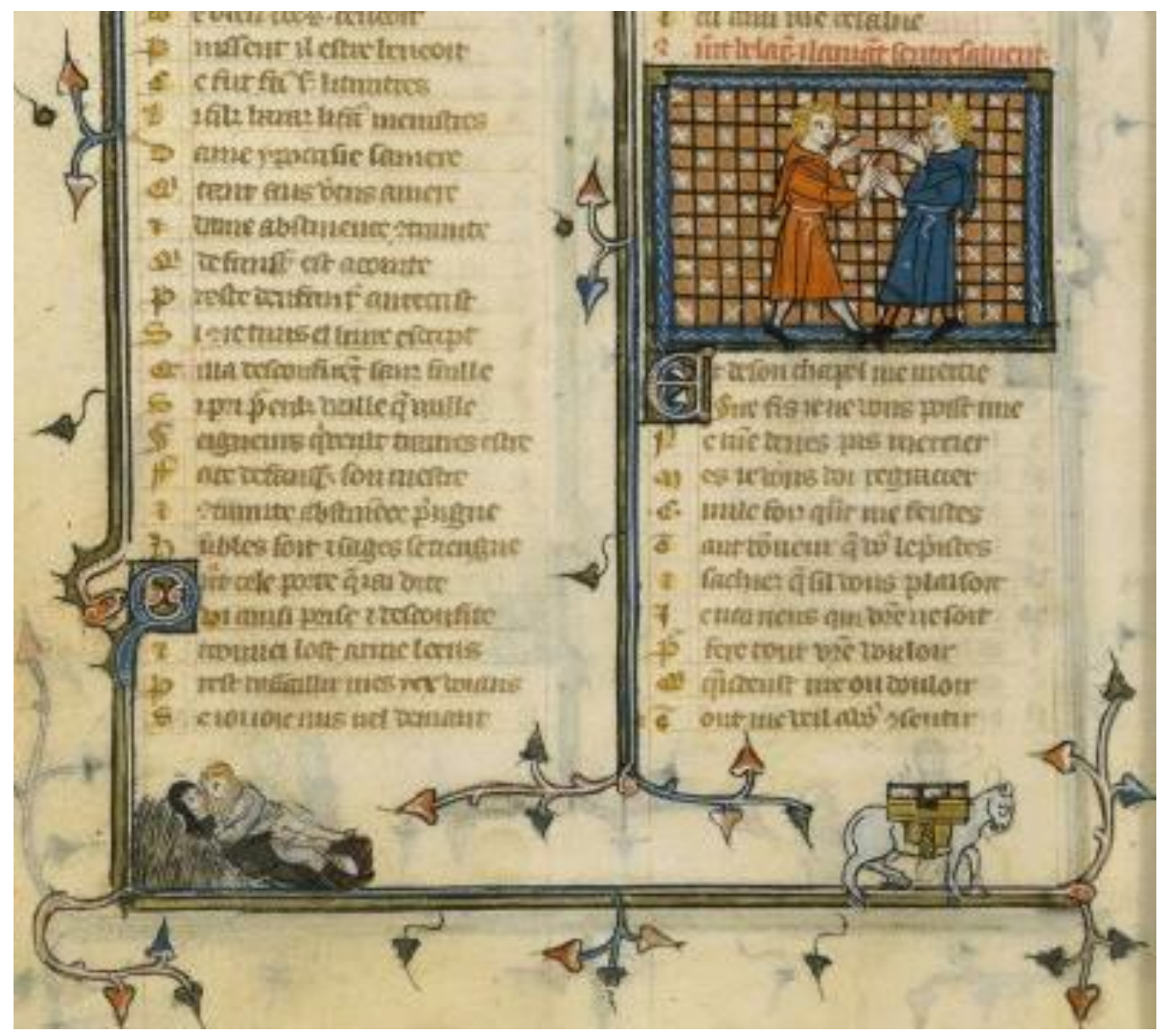

Illustration 7

JEANNE DE MontBaSton, « Nonette en bonne compagnie », GUILLAUME DE LORRIS et JEAN DE MEUn, Le Roman de la Rose, fin du XIV ${ }^{\mathrm{e}}$ siècle, Paris, BnF, f. fr. 25526, f $\mathrm{f}^{\circ} 111 \mathrm{v}^{\circ}$. 


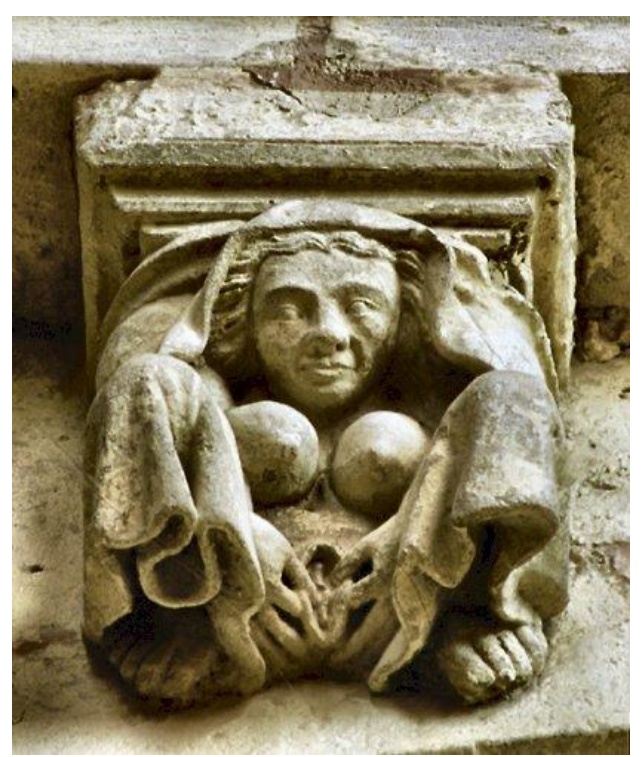

Illustration 8

Modillon de l'église Sainte Radegonde, $\mathrm{XIII}^{\mathrm{e}}$ siècle, Poitiers.

Crédit photo : Anthony Weir.

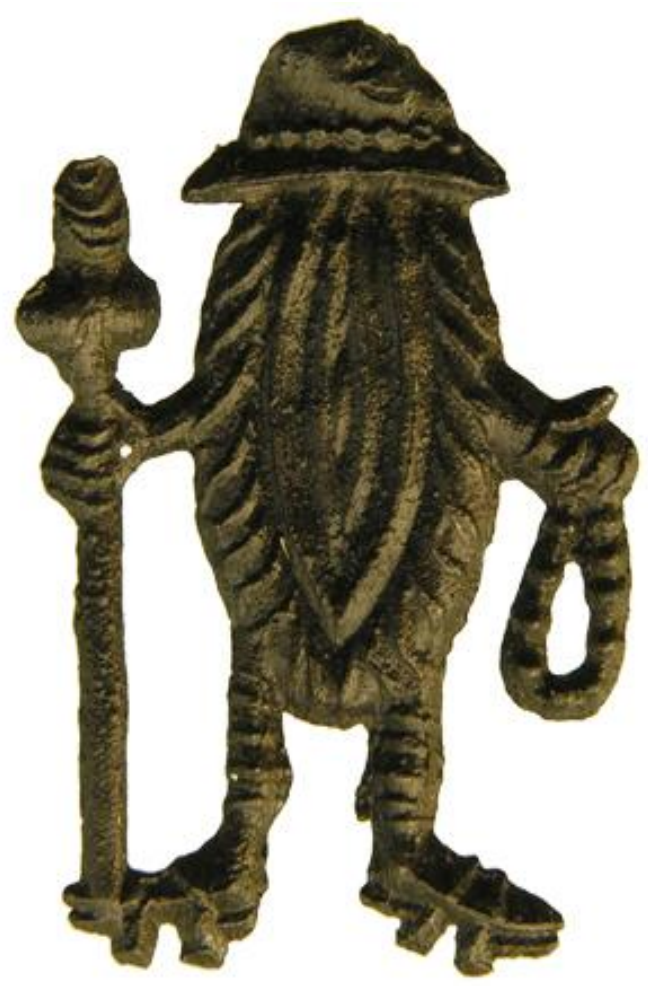

Illustration 10

Enseigne, 1375-1425,

Reimerswall, collection H. J. E.

Beuningen, Inv. 2184.

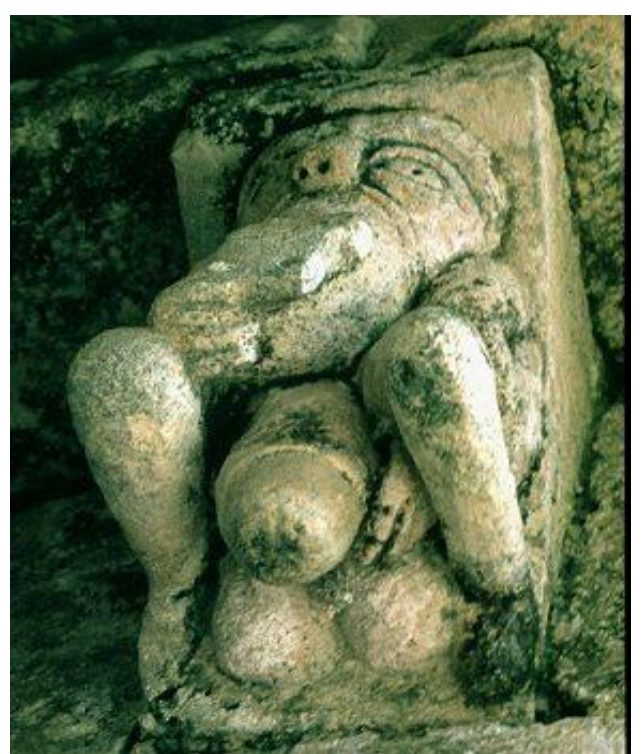

Illustration 9

Modillon de l'église de Champagnolles, $\mathrm{XIII}^{\mathrm{e}}$ siècle.

Crédit photo : Anthony Weir.

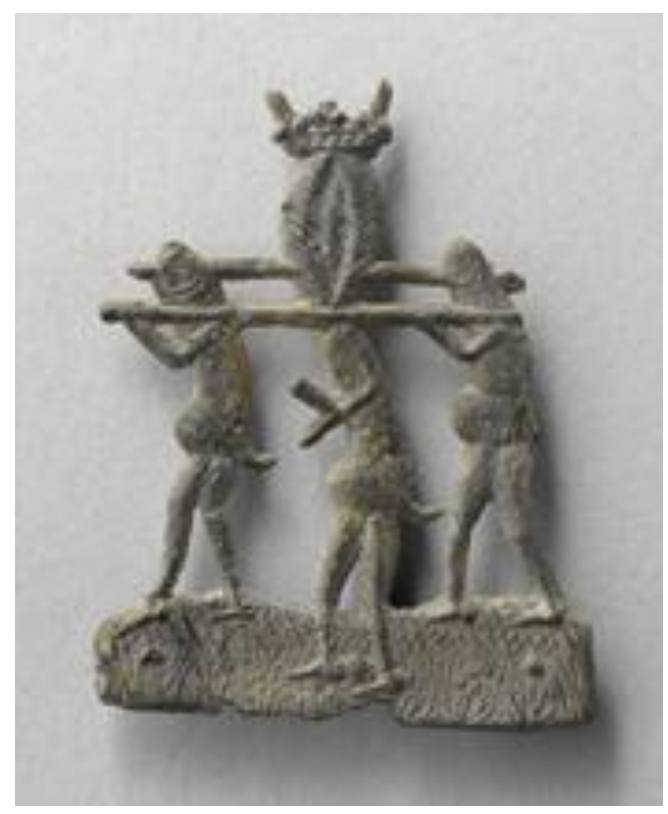

Illustration 11

Enseigne, $\mathrm{XV}^{\mathrm{e}}$ siècle, Paris, Musée national du Moyen Âge. 